\title{
Effect of facial cooling and cold air inhalation on sympathetic nerve activity in men
}

\author{
Silke Heindl, Jan Struck, Peter Wellhöner, Friedhelm Sayk, Christoph Dodt* \\ Department of Internal Medicine I, University of Luebeck, Ratzeburger Allee 160, 23538 Luebeck, Germany
}

Accepted 19 May 2004

\begin{abstract}
In nine healthy subjects, cold stimuli were administered to the forehead and hand, to the oral and nasal cavities via ice cubes and to the bronchial system via inhalation of cold air $\left(-25^{\circ} \mathrm{C}\right)$. Blood pressure, heart rate and muscle sympathetic nerve activity (MSNA) from the peroneal nerve were recorded. MSNA expressed as total activity increased during cold air inhalation, cooling of the forehead $(P<0.001$, ANOVA), hand and mouth $(P \leq 0.05)$, paralleled by a rise in blood pressure during cold air inhalation and cooling of the forehead and hand $(P<0.01)$. Cooling of the forehead provoked a faster increase of MSNA expressed as total activity $(P<0.05)$ and higher levels of diastolic blood pressure $(P=0.05)$ compared with cooling of the hand. Bradycardia was observed only during cooling of the nasal cavity $(P<0.001)$ and the forehead $(P<0.05)$. It is concluded that cooling of the skin and mucous membranes of the tracheobronchial tract elicits sympathetically mediated hemodynamic adaptations, probably via stimulation of cold-sensitive afferents.
\end{abstract}

(C) 2004 Elsevier B.V. All rights reserved.

Keywords: Mammal; Humans; Nervous system; Sympathetic; Airway cooling; Temperature; Facial cooling; Cold air inhalation; Upper airways; Cold exposure

\section{Introduction}

Cold stimulation of the skin elicits marked cardiovascular reflexes in which sympathetic activation plays an important role. Muscle sympathetic nerve activity (MSNA) consistently increases during immersion of parts of the skin in ice water and the cold pressor test (CPT) is well established as stimulation test in research settings. Immersing the hand in ice water evokes a marked increase in MSNA and blood pres-

\footnotetext{
* Corresponding author. Tel.: +49 4515002329 ; fax: +494515002328 .

E-mail address: christoph.dodt@medinf.mu-luebeck.de (C. Dodt).
}

sure. These changes are accompanied by a minor, albeit significant increase in heart rate (Victor et al., 1987; Fagius et al., 1989). The response is probably evoked either by afferent impulses of cold receptors alone or by a combination of afferent impulses from pain and cold receptors (Fagius et al., 1989). The effect of pain receptors on sympathetic activation is known to depend on the stimulated area and seems to be strongest in the face.

The role of facial receptors in cardiovascular reflexes has also been investigated before (Fagius and Sundlöf, 1986; Nordin and Fagius, 1995). Various types of noxious stimuli to the trigeminal region resulted in a reflex pattern characterized by rises in MSNA and blood pressure and by bradycardia. This 
reflexive pattern of both sympathetic and parasympathetic activation has also been described during immersion of the face in cold water and is called diving response. It is initiated by impulses from cold receptors and probably mechanoreceptors of the face and reinforced by the effects of apnoea (Fagius and Sundlöf, 1986).

At present, there is little information on the autonomic effects of localized skin cooling or cooling of the mucous membranes of the respiratory tract in humans. The face and the respiratory tract constitute the body regions exposed to cold stimuli under physiological conditions in humans. Thus, the sympathetic reflex response to cooling of these body regions is of particular interest. Therefore, we determined hemodynamic parameters and MSNA response in healthy subjects after inhalation of cold air $\left(-25^{\circ} \mathrm{C}\right)$. We also compared the effects on cardiovascular reflex adjustments during cooling of the nasal and oral mucous membranes and of the forehead with the response of cooling of the back of the hand. During the different manoeuvres MSNA was recorded from the peroneal nerve to assess sympathetic activity. The aim of the study was to find out differences in the adjustments of MSNA, blood pressure and heart rate during localized cooling of the skin and the respiratory tract.

\section{Methods}

\subsection{Subjects}

We included male subjects aged 18-35 years with normal body mass index. All subjects were healthy, non-smokers and were not taking any medications. They were asked to abstain from alcohol the day before the experiment and to abstain from caffeine for $\geq 6 \mathrm{~h}$ before the start of the experiment. The study was approved by the local ethics committee, and all subjects gave their written informed consent.

\subsection{Measurements}

Sympathetic activity was measured using microneurographic recordings of efferent MSNA in the peroneal nerve as described previously (Dodt et al., 2000; Heindl et al., 2001). After mapping the course of the peroneal nerve around the head of the fibula with tran- scutaneous electrical stimulation, a Tungsten microelectrode was inserted into the nerve. A reference electrode was inserted subcutaneously $3 \mathrm{~cm}$ apart. When a muscle nerve fascicle had been identified by electrical stimulation through the microelectrodes, small adjustments of the intraneural electrode were made until MSNA was encountered. The criteria for an adequate recording of MSNA were the following: (1) electrical stimulation through the intraneural electrode elicited involuntary muscle contractions but not cutaneous paresthesia, (2) stretching the muscle innervated by the impaled fascicle elicited afferent mechanoreceptor discharges, but slight tactile stimuli within the distribution area did not evoke afferent impulses, (3) Valsalva manoeuvre but not arousal stimuli increased burst frequency and (4) the spontaneous activity occurred in intermittent and pulse synchronous bursts (Delius et al., 1972; Vallbo et al., 1979). The nerve signals underwent amplification (50000 times), bandpass filtering (band width of 700-2000 Hz), and passed through a resistance-capacitance integrating network with a time constant of $0.1 \mathrm{~s}$, providing a mean voltage display of sympathetic nerve activity.

The ECG was recorded with standard chest leads. Blood pressure was measured oscillometrically (Welch Allyn Tycos) $10 \mathrm{~min}$ after the subjects had resumed the supine position at the beginning of the experiment. Relative blood pressure changes were monitored with blood pressure measurements from a finger with the hand resting at the level of the heart, with the volume-clamp technique (Finapres, Ohmeda Monitoring Systems, Englewood, Colorado, USA). Respiratory movements were monitored with a strain gauge strapped around the chest with a rubber band to control for inadvertent apneas and irregular breathing, which are known to affect MSNA.

Analog signals of all parameters (mean voltage neurogram, ECG, blood pressure, respiration) were digitized online with a sampling rate of $200 \mathrm{~Hz}$ (CED 1401, Cambridge Electronic Design, Cambridge, England) and stored on a computer disk. Signals were also printed out with a Nihon Kohden 4421 Neurofax.

\subsection{Protocol}

Subjects were studied in the morning, $2 \mathrm{~h}$ after a low-calorie breakfast free of caffeine-containing beverages. The microelectrodes for nerve recordings, the 
ECG electrodes, the blood pressure cuff and the respiratory band were positioned and the subjects rested in a comfortable supine position. After a satisfactory nerve signal had been obtained, the protocol started with a 5-min recording period while the subjects were spontaneously breathing room air at complete rest (baseline).

A respiratory heat exchange system (RHES plus, Jaeger Toennies, Germany) was used for inhalation of cold air. Prior to the cold air inhalation the subjects were made familiar with the procedure: they started to breathe room air through the mouthpiece of the heat exchange system but were not connected to the heat exchanger. During this period, their breathing frequency at rest was determined.

Then, the subjects were connected to the heat exchange system and a recording period of $2.5 \mathrm{~min}$ was started while the subjects were breathing air at room temperature through the heat exchanger (breathing room air with a mouthpiece). Then the air was cooled down to $-25^{\circ} \mathrm{C}$ and subjects ventilated through the heat exchange system for additional 5 min (cold air inhalation). To avoid irregular breathing or hyperventilation, respiratory rate was kept at the predetermined frequency by a metronome during these two manoeuvres.

After a resting period of $10 \mathrm{~min}$, the subjects were successively asked to keep an ice cube in the mouth, to put little plastic cups filled with ice in the nostrils and to put an ice-filled plastic bag of standardized size $(12 \mathrm{~cm} \times 18 \mathrm{~cm})$ on the forehead and on the back of the left hand, respectively. Each manoeuvre was performed for $2.5 \mathrm{~min}$ while the subjects were breathing regularly and avoiding isometric muscle contraction. The order of the manoeuvres was randomized and they were separated by $10 \mathrm{~min}$ rest. The minute preceding each manoeuvre was recorded for further analyses (preceding rest period).

\subsection{Data analysis}

MSNA recordings were analysed by the same observer (S.H.) who was blinded regarding the manoeuvres. Sympathetic bursts were visually identified and further quantified with the aid of a software developed for the analysis of sympathetic nerve activity and related cardiovascular parameters (T. Karlsson, Department of Clinical Neurophysiology, University of
Gothenburg, Sweden). This analysis software allows the determination of the area of the bursts marked during the analysis and subsequently calculates the total nerve activity (area under the curve $\times$ burst number/min, arbitrary units), heart rate and blood pressure on a beat-to-beat basis during the experimental periods. The Finapres method reliably measures relative blood pressure changes while the absolute blood pressure values are less reliably obtained. Therefore, all blood pressure values were expressed as percentage of the baseline values.

The following periods were evaluated: $5 \mathrm{~min}$ of baseline; $2.5 \mathrm{~min}$ of breathing room air with a mouthpiece; $5 \mathrm{~min}$ of cold air inhalation; $2.5 \mathrm{~min}$ of the manoeuvres ice-in-mouth, ice-in-nose, ice-on-forehead and ice-on-hand as well as the 1 min preceding each cooling manoeuvre.

For analysis, MSNA and hemodynamic parameters of the various manoeuvres were averaged for $30 \mathrm{~s}$ periods and compared to the directly preceding resting period of 1 min duration. The effects of cold air inhalation were evaluated by analysis of variance (ANOVA) with the repeated measures factor time. The effects of the cooling manoeuvres performed on different face regions and the dorsum of the hand were evaluated by ANOVA with the repeated measures factor time and the factor cold modality. Statistical differences between the baseline period and the single values during the manoeuvres were analysed with the Duncan's post hoc test when the overall ANOVA indicated significance. The null hypothesis was rejected at a $P$-value $<0.05$. All results are presented as mean \pm standard error of the mean (S.E.M.).

\section{Results}

The cooling manoeuvres on the skin and mucous membranes of the oral and nasal cavity were performed on nine healthy subjects. Eight subjects additionally participated in the room air and cold inhalation manoeuvres. Fig. 1 shows the experimental records from one subject during each of the cooling manoeuvres.

When comparing the baseline values with the values obtained from the $1 \mathrm{~min}$ intervals preceding each cooling manoeuvre, MSNA (expressed as bursts per minute and total activity) or heart rate did not change 
prior cold air inhalation

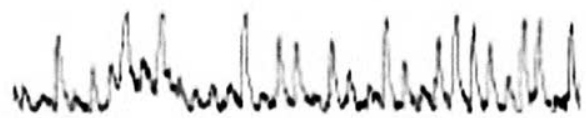

prior ice-in-mouth

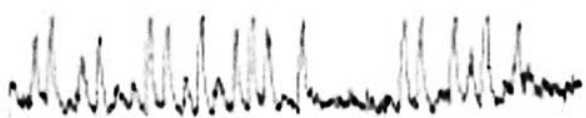

prior ice-in-nose

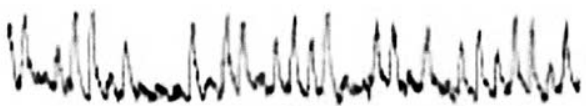

prior ice-on-hand

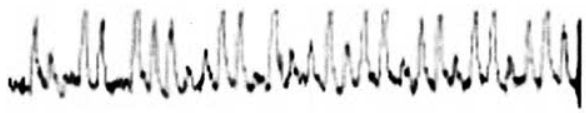

prior ice-on-forehead

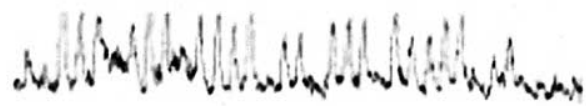

cold air inhalation $\left(-25^{\circ} \mathrm{C}\right)$

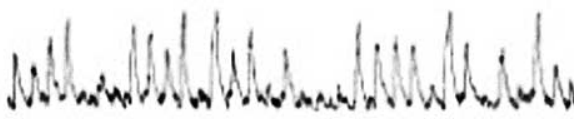

ice-in-mouth

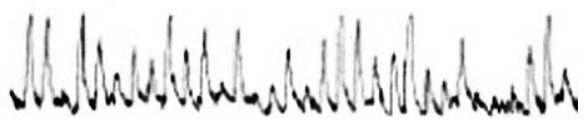

ice-in-nose

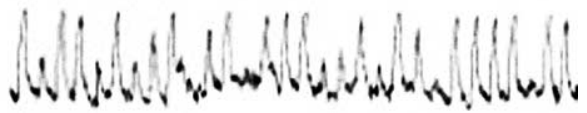

ice-on-hand

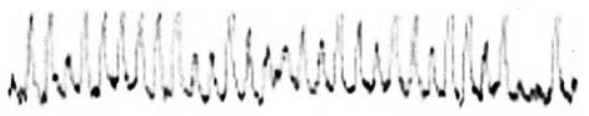

ice-on-forehead
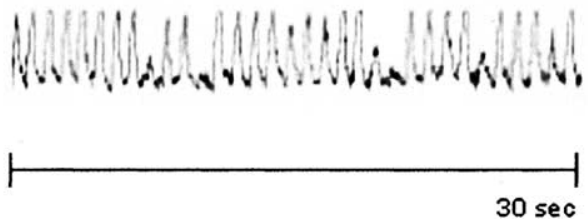

Fig. 1. Experimental records during cold air inhalation and the topical cooling manoeuvres. Mean voltage records of MSNA obtained while the subject was breathing room air with a mouthpiece are compared with the MSNA records during cold air inhalation. The MSNA records during the corresponding preceding rest periods are each followed by the records during cooling of the forehead, hand, mouth and nose. All records were obtained from the same subject, during one experimental session.

over the length of the whole experiment. However, there was an increase in systolic blood pressure by 9.1 $\pm 3.5 \%(P<0.001$, ANOVA $)$ and in diastolic blood pressure by $7.5 \pm 3.5 \%(P<0.01$, ANOVA) when comparing the $1 \mathrm{~min}$ interval preceding the last cooling manoeuvre with the baseline values at the beginning of the microneurographic recording. We will therefore compare all values obtained during the cooling ma- noeuvres with the corresponding, that is the directly preceding rest period in further analyses.

\subsection{Inhalation of cold air}

Breathing room air with a mouthpiece did not alter MSNA expressed as bursts per minute, heart rate and systolic blood pressure compared with the base- 
line values $(P=$ n.s., ANOVA). However, there was a decrease of sympathetic total activity $(P<0.001$, ANOVA $)$ and of diastolic blood pressure $(P<0.05$, ANOVA) during breathing with a mouthpiece. Because of this effect of controlled mouthpiece breathing, values obtained during inhalation of cold air were compared with the values during breathing room air with a mouthpiece in further analyses.

Inhalation of cold air $\left(-25^{\circ} \mathrm{C}\right)$ caused significant increases in MSNA (expressed as bursts/min, $P=0.05$, ANOVA and expressed as total activity, $P<0.001$, ANOVA). Systolic and diastolic blood pressure were also significantly elevated $(P \leq 0.001$, ANOVA), while heart rate was not affected (Fig. 2).

\subsection{Topical cooling of the skin and of the oral and nasal cavity}

Ice-on-forehead and ice-on-hand provoked a considerable increase in MSNA (expressed as bursts per minute and total activity). When comparing the two manoeuvres, the increase in MSNA expressed as total activity was faster during ice-on-forehead than during ice-on-hand $(P<0.05$, ANOVA) with a significant difference during the first $30 \mathrm{~s}$ of the manoeuvre $(P<0.01$, Fig. 3). Ice-in-mouth increased MSNA (expressed as bursts/min and total activity) while ice-in-nose did not influence MSNA at all (Fig. 4).

The changes in MSNA were paralleled by the blood pressure changes during all cooling manoeuvres: both systolic and diastolic blood pressure increased during ice-on-forehead and ice-on-hand (Fig. 3). Ice-in-mouth caused an increase in systolic blood pressure and a trend towards higher diastolic blood pressure, while ice-in-nose did not change systolic or diastolic blood pressure (Fig. 4). When comparing the diastolic blood pressure during the different cooling manoeuvres, overall ANOVA showed significant effects for the repeated measures factor time and factor cold modality as well as significant interaction between time and cold modality. When comparing ice-on-forehead and ice-on-hand, the increase in diastolic blood pressure was more pronounced during ice-on-forehead than during ice-on-hand $(P=0.05$, ANOVA) with a significant difference $90 \mathrm{~s}$ after the start of the manoeuvre $(P<0.05$, Fig. 3$)$.

While ice-in-nose and ice-on-forehead provoked a significant fall in heart rate, there was no change in heart rate during the manoeuvres ice-in-mouth and ice-on-hand (Figs. 3 and 4). When comparing the heart rate during the manoeuvres ice-in-nose and ice-on-forehead, ANOVA did not reveal any significant effect for the factor cold modality nor any significant interaction between time and cold modality.

The changes in bursts per minute, diastolic blood pressure and heart rate during the first $60 \mathrm{~s}$ of each manoeuvre are summarized in Fig. 5. During any of the cold stimulation manoeuvres, neither consistent changes in the respiratory pattern nor individual gasps occurred that may have influenced the results.

\section{Discussion}

The present study confirms that a pronounced increase in sympathetic discharge to the skeletal muscle is induced by different cold stimuli in young healthy humans. The new finding is that the response strongly depends on the body region which is exposed to the cold stimulus and is strongest when applied to the face (i.e. forehead). Furthermore, it can also be observed during inhalation of cold air.

\subsection{Localized cooling of the skin}

Cooling of the skin of the forehead and the back of the hand evoked different cardiovascular adjustments. Both manoeuvres induced strong increases in MSNA and in systolic and diastolic blood pressure. The increase in MSNA occurred faster and was initially more pronounced during forehead cooling than during hand cooling. Also, diastolic blood pressure clearly showed a stronger increase during cooling of the forehead. In contrast, heart rate demonstrated an initial decrease during ice-on-forehead but remained constant during ice-on-hand.

The increase in MSNA and blood pressure during hand cooling is a well known effect and this response has been used as CPT in several studies as a standardized baroreflex-independent stimulatory manoeuvre of the MSNA (Victor et al., 1987; Fagius et al., 1989). The parallel increase in blood pressure and MSNA during the CPT has led to the conclusion that a change of the baroreflex inhibitory level was induced by some extraordinary input operating during the manoeuvre (Fagius et al., 1989). During the CPT, 

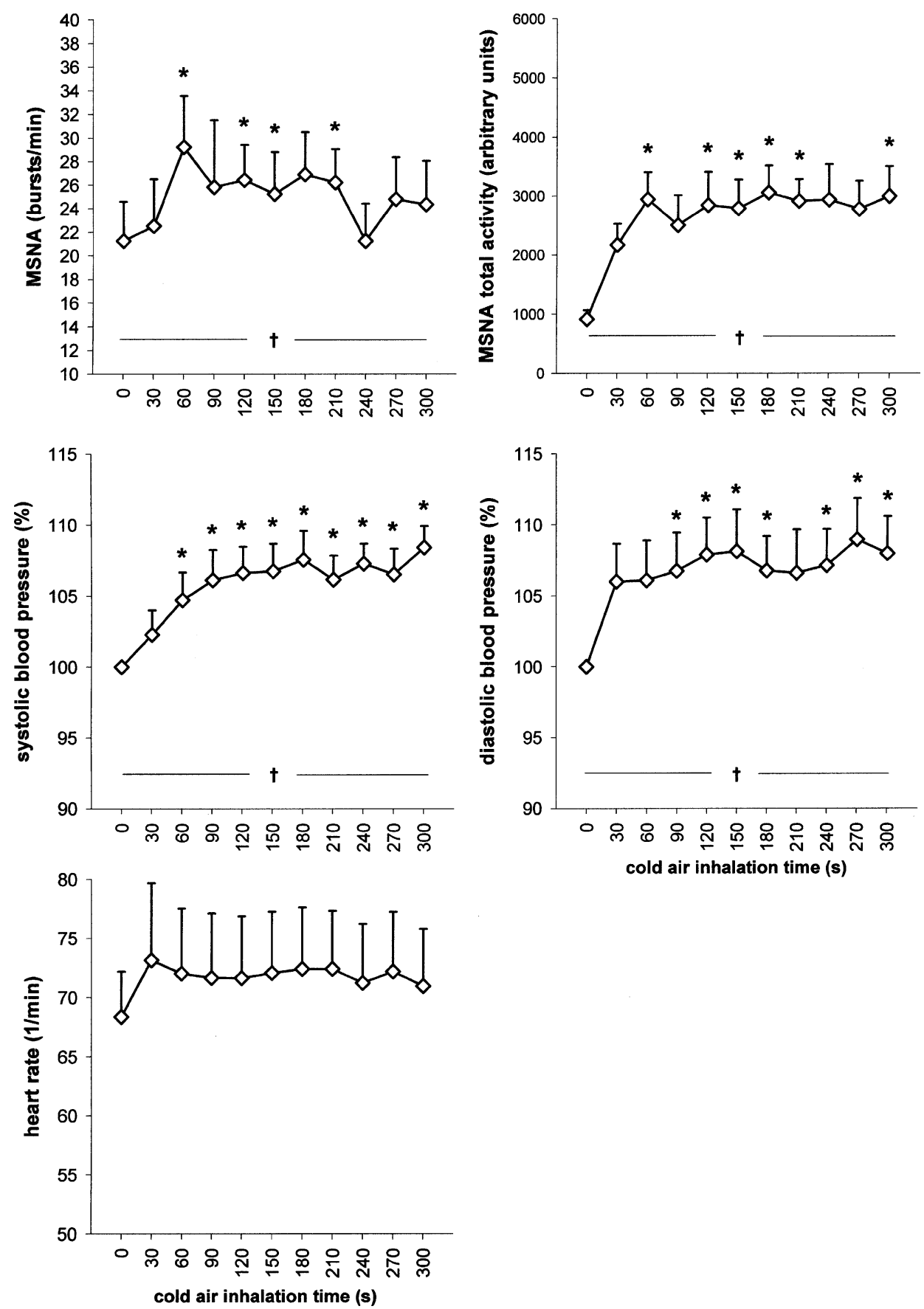

Fig. 2. Effects of cold air inhalation. MSNA (expressed as bursts/min and total activity), systolic and diastolic blood pressure as well as heart rate during a 5-min period of inhalation of cold air $\left(-25^{\circ} \mathrm{C}\right)$. The values obtained during cold air inhalation were compared with the values obtained during a 2.5 -min period of breathing room air with a mouthpiece (cold air inhalation time $0 \mathrm{~s}$ ). Values are given as mean \pm S.E.M. ${ }^{\dagger} P \leq 0.05$ vs. room air $(P=0.05$ for MSNA bursts/min, $P<0.001$ for MSNA total activity, systolic and diastolic blood pressure; ANOVA). ${ }^{*} P<0.05$ (post hoc test). 

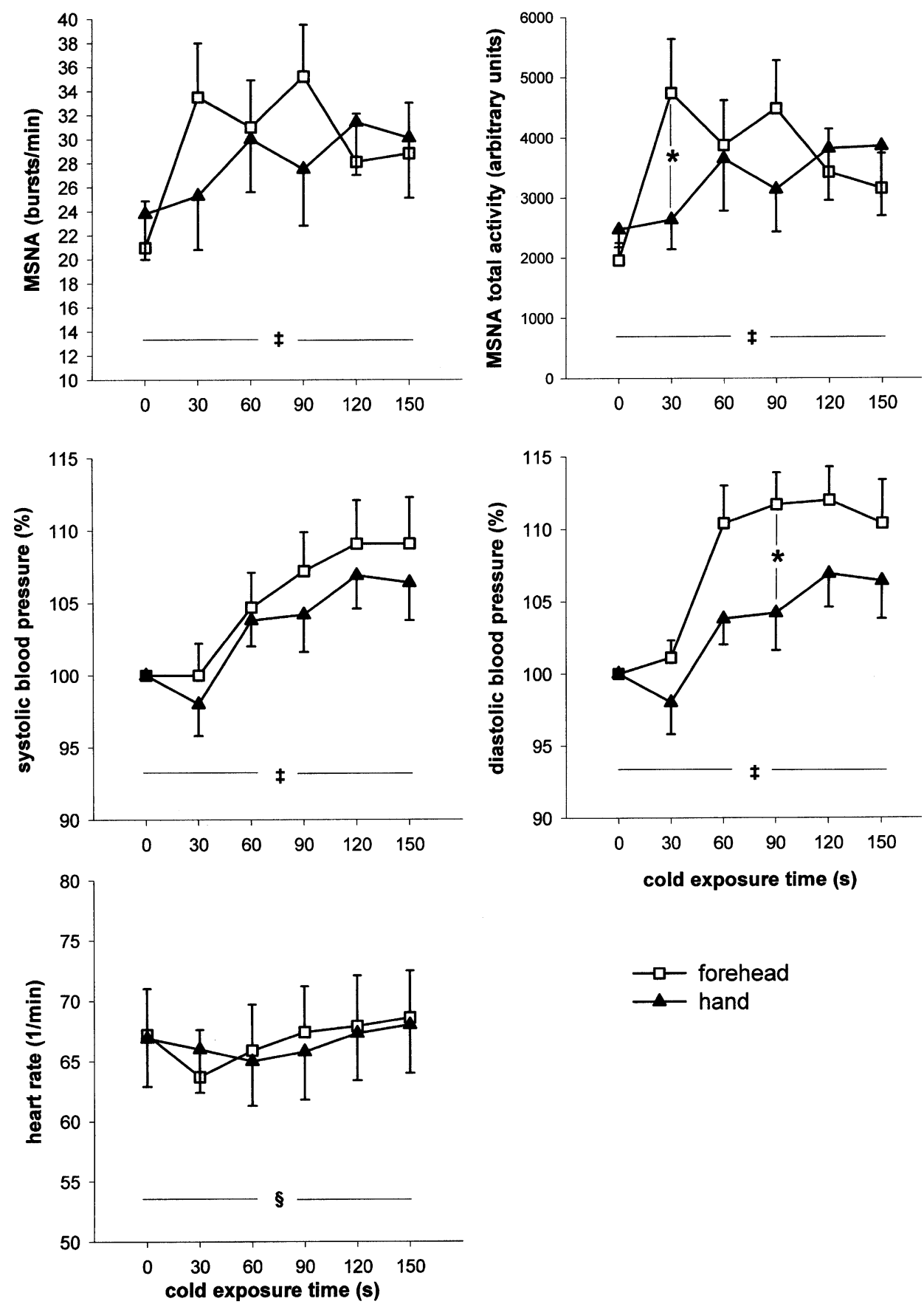

Fig. 3. Effects of localized skin cooling. MSNA (expressed as bursts/min and total activity), systolic and diastolic blood pressure as well as heart rate during cooling of the forehead and dorsum of the hand. The values obtained during each manoeuvre were compared with the values obtained during the preceding rest period. Values are given as mean \pm S.E.M. (\$) Significant effect of both ice-on-forehead and ice-on-hand (ANOVA); (§) significant effect of ice-on-forehead (ANOVA) (*) significant difference between ice-on-forehead vs. ice-on-hand (post hoc test). 

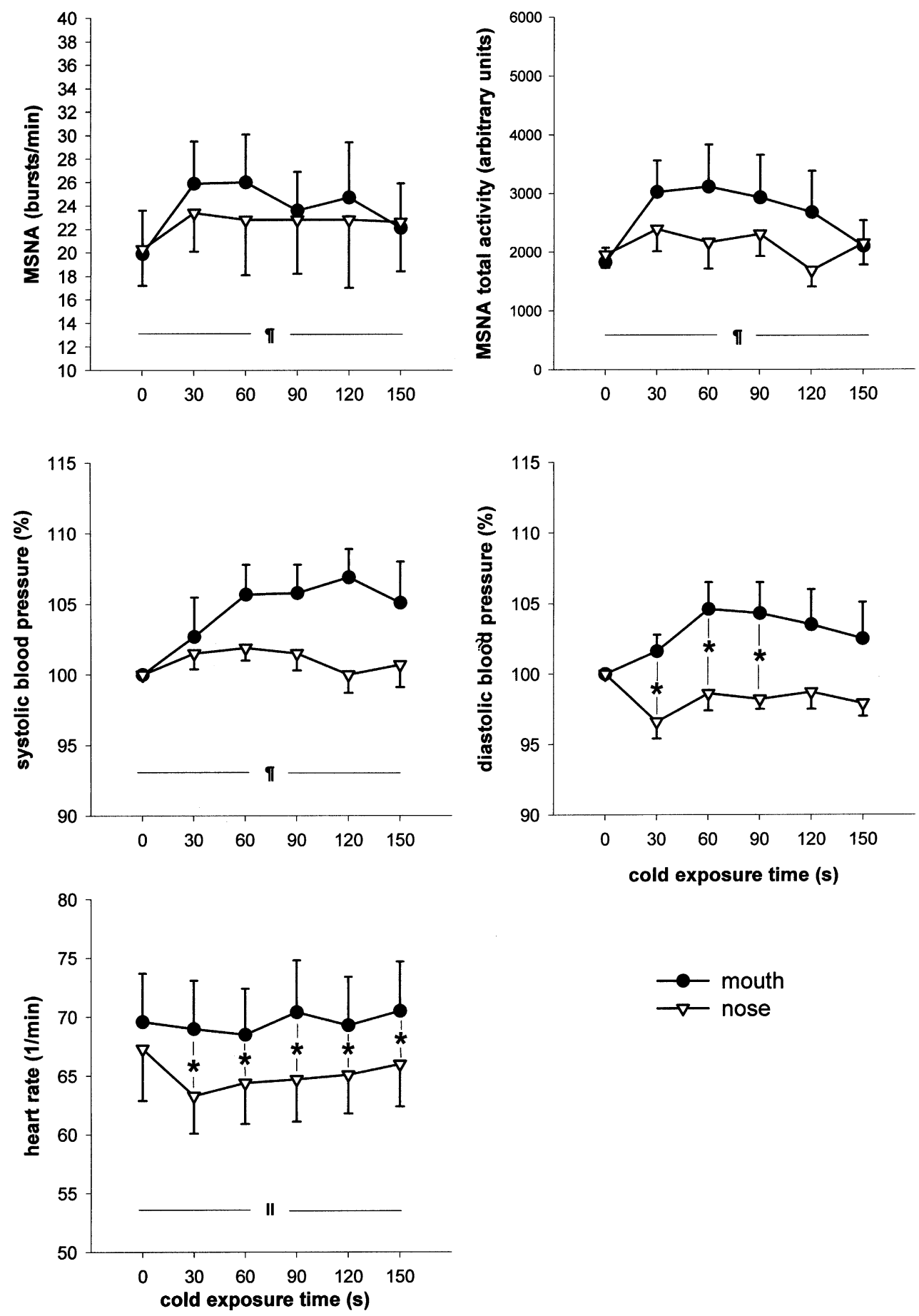

Fig. 4. Effects of cold stimuli to the oral and nasal cavities. MSNA (expressed as bursts/min and total activity), systolic and diastolic blood pressure as well as heart rate during cooling of the oral and nasal cavities. The values obtained during each manoeuvre were compared with the values obtained during the preceding rest period. Values are given as mean \pm S.E.M. (II) Significant effect of ice-in-mouth (ANOVA); (\|) significant effect of ice-in-nose (ANOVA) (*) significant difference between ice-in-mouth vs. ice-in-nose (post hoc test). 


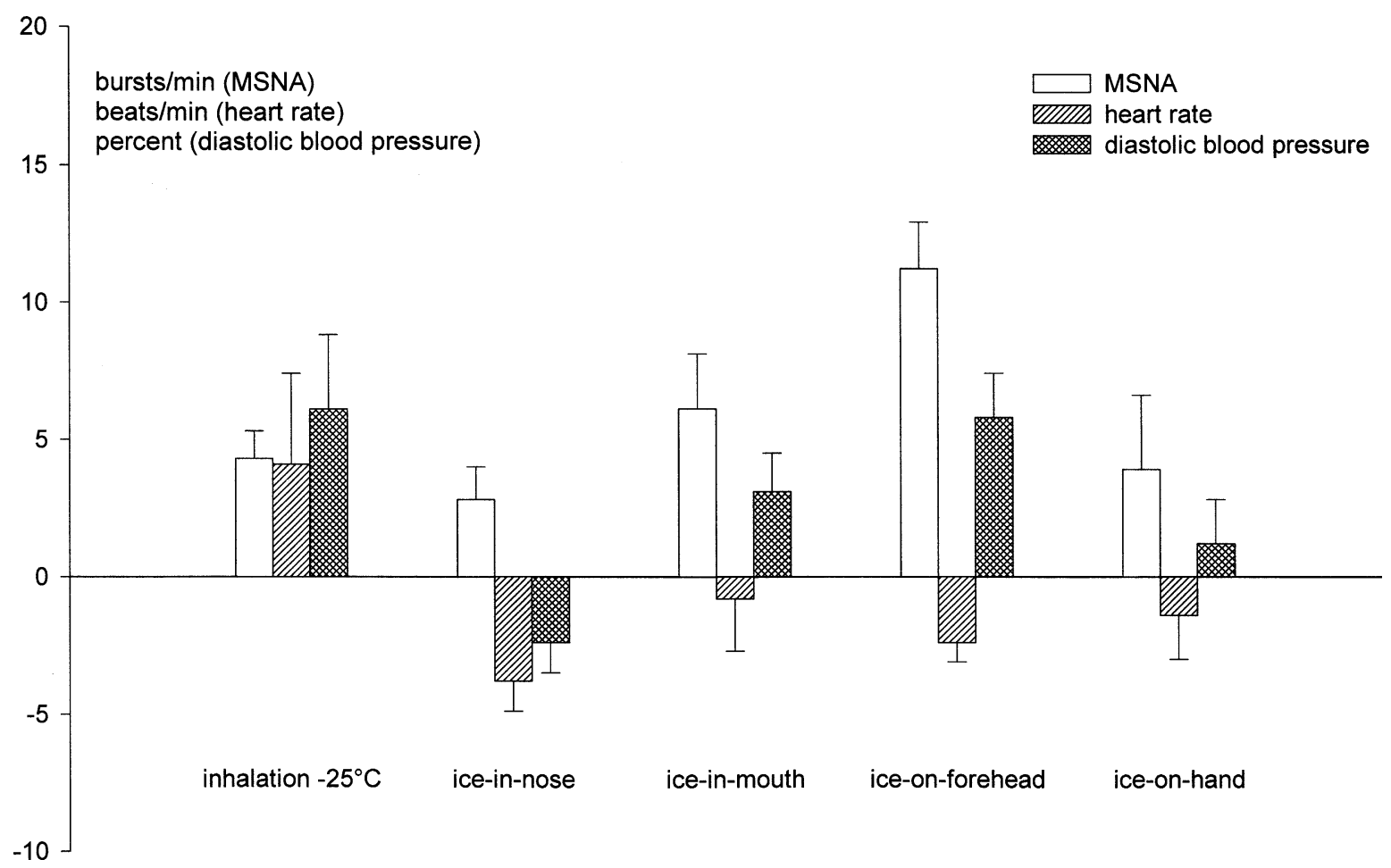

Fig. 5. Summary of the adjustments of MSNA and hemodynamic parameters during the different cooling manoeuvres. Changes of MSNA (expressed as bursts/min), heart rate (beats/min) and diastolic blood pressure (in percent) during the different cold exposure tasks. Changes of MSNA, heart rate and diastolic blood pressure are calculated as the difference between the mean value during the first minute of each manoeuvre and the value obtained during the preceding rest period. All values are given as mean \pm S.E.M.

the increase in MSNA is most likely evoked by a combined pattern of afferent impulses from pain and cold receptors to central nervous autonomic centers (Fagius et al., 1989). Both excitation of low-threshold, cold-sensitive receptors (A $\delta$ and $\mathrm{C}$ fibres) and of high-threshold, cold-sensitive nociceptive fibres (primarily $\mathrm{C}$ fibres) have been postulated during cooling of the hand (Kregel et al., 1992).

Usually, the cardiovascular response to the CPT includes a slight increase in heart rate (Victor et al., 1987; Fagius et al., 1989), which has not been observed in the present experiment of localized hand cooling. However, it has been suggested that the acceleration of heart rate is related to the pain sensation which is induced by the immersion of the whole hand in ice water (Victor et al., 1987). Therefore, it seems likely that the difference in heart rate response is due to the smaller number of pain fibres stimulated during localized cooling of the dorsum of the hand.
The differences in autonomic response observed during localized cooling of the hand and forehead in our study are remarkable because stimulus intensity and area of exposed skin were equal in both manoeuvres. These differences may partly result from the specific distribution of cold and pain receptors and mechanoreceptors in the skin of the forehead compared with the skin of the dorsum of the hand. Obviously, the skin of the forehead represents an important region for the adaptation of the cardiovascular system to cold exposure.

The changes observed during ice-on-forehead are consistent with the reflex pattern observed during various types of stimuli to the trigeminal region previously described. Pressure applied to the supraorbital ridge, electrical stimulation of the supraorbital nerve, instillation of soap solution in the eye and immersion of the face in cold water all caused or tended to cause bradycardia and an increase in 
MSNA and blood pressure (Fagius and Sundlöf, 1986; Nordin and Fagius, 1995). Very simple manoeuvres like placing an ice-bag on the forehead or directing a stream of cold air $\left(0-10^{\circ} \mathrm{C}\right)$ towards the face also evoked similar hemodynamic adjustments (Hayward et al., 1976; Allen et al., 1992; LeBlanc and Mercier, 1992; Trouerbach et al., 1994; Walsh et al., 1995). This response pattern resembles the diving response which is initiated by immersion of the face in water. During the classic diving response, bradycardia results from increased parasympathetic outflow to the cardiac pacemaker and the increase in MSNA causes vasoconstriction and a reduction in limb blood flow (Fagius and Sundlöf, 1986). Cold receptors and probably mechanoreceptors of the face are involved in the initiation of the increase in MSNA, and the impulses from facial receptors and effects of apnoea seem to act by mutual reinforcement (Fagius and Sundlöf, 1986). Thus, cold exposure to the forehead not only enhanced sympathetic nerve traffic to the muscle vascular bed but probably also increased parasympathetic tone to the heart in our study.

\subsection{Tracheobronchial, oral and nasal cooling}

Not only cooling of the facial skin but also inhalation of cold air induced a strong response of the cardiovascular system - with an increase in MSNA, heart rate and blood pressure. Restriction of the cold exposure to the oral cavity or the nasal cavity elicited different responses. Ice-in-mouth induced an increase in MSNA and blood pressure while heart rate remained unaffected. Cooling of the nasal cavity decelerated heart rate but did not change MSNA and blood pressure. Thus, our results clearly show a specific cardiovascular response pattern depending on the part of the respiratory system exposed to the cold stimulus. The reason for these differential cardiovascular responses is a matter of speculation. However, the distribution of sensory afferents and their specific mode of excitation is likely to explain the results.

It is known that the tracheobronchial and nasal mucosa is supplied with various types of sensory afferents. In anesthetized dogs, laryngeal receptors are found that operate as thermoreceptors activated by cooling (Sant' Ambrogio et al., 1985). In the human larynx, cold, pressure, drive, irritant and $\mathrm{C}$ fibre receptors have been described (Widdicombe, 2001).
There also is evidence of osmosensitive receptors in the canine airway mucosa (Baile et al., 1987) as well as in the human nasal mucosa (Togias et al., 1990; Fontanari et al., 1996). Slowly and rapidly adapting stretch receptors, C fibre receptors and receptors in neuroepithelial bodies have been identified in the human trachea and bronchi, the reflex actions of the latter being unclear (Widdicombe, 2001).

Undoubtedly, the nose plays a special role in the autonomic nervous regulation of protective reflexes, e.g. the diving response, sneeze and sniff reflexes. It shows a highly complex innervation including parasympathetic, sympathetic, sensory/afferent and somatic motor nerves (Widdicombe, 1986). The distribution density of cold and warm receptors in the nasal vestibule (that part of the nasal cavities lined with skin) is higher than in the facial skin. Interestingly, no thermoreceptors are found in the nasal cavum (that part of the nasal cavities lined with respiratory epithelium) (Jones et al., 1989). This fact rises the question whether the bradycardia found during the manoeuvre ice-in-nose in our study was caused by the activation of cold receptors in the skin of the nasal vestibule rather than in the mucosa of the nasal cavity itself.

During the cold air inhalation manoeuvre performed in our study, both oral and tracheobronchial structures were exposed to the cold and/or dry stimulus and it remains unresolved which afferent nerves transmitted the stimulus to the central nervous system. It can also not be excluded that during the ice-in-mouth manoeuvre cooling and/or drying of the larynx or the upper gastrointestinal tract occurred which could have affected MSNA and blood pressure. However, our study shows that those nerve endings or receptors which are excited during cold exposure of the tracheobronchial tract are able to induce sympathetically-mediated cardiovascular adaptations independent from facial receptors. Particularly inhalation of cold air represents a strong and sustained stimulus of the sympathetic outflow to the muscle vascular bed.

The finding that cooling of the nasal mucosa causes net bradycardia is quite remarkable and can be interpreted as an isolated activation of the parasympathetic system. This may have clinical relevance since nasal breathing but not oral breathing of cold dry air ( -4 and $-10^{\circ} \mathrm{C}$ ) induced bronchoconstriction in healthy subjects probably via parasympathetic activation. However, bronchoconstriction did not occur after 
nasal anesthesia suggesting that activation of cold and/or osmosensitive receptors in the nasal mucosa induced protective bronchoconstrictor responses in normal subjects (Fontanari et al., 1996). As we did not investigate parasympathetic activity or airway resistance in our study, the origin of bradycardia during nasal cooling remains uncertain.

\subsection{Limitations of the study}

We can not specify the neural afferents which sense cold stimuli and trigger the observed sympathoneural reflexes and this is the major limitation of the study. For example, previous work has suggested that osmoreceptors of the nasal and airway mucosa are particularly important for cold sensation (Baile et al., 1987; Togias et al., 1990; Fontanari et al., 1996). A transient change in osmolarity in the respiratory tract fluid could influence bronchial vascular smooth muscle tone either by direct osmotic effects or through local release of mediators or by stimulating afferent receptors that would result in a reflex vasodilatation via parasympathetic or sympathetic pathways. During inhalation of cold air, by obstructing the nasal cavity with ice cups or by keeping an ice cube in the mouth these receptors may have been affected and could have altered cardiovascular adaptation to cold exposure.

Alterations of ventilation or blood gases may also have caused modulations in MSNA during the different cold stimuli (Hardy et al., 1994; Morgan et al., 1995). In our study, respiratory movements were monitored with a strain gauge strapped around the chest with a rubber band to control the inadvertent apneas and irregular breathing. Also, we determined the respiratory rate prior to the cold inhalation through the heat exchange system and trained the subjects to keep their breathing rhythm throughout the experiment with the help of a metronome. However, the experimental setting and the resistance of the tubes of the heat exchange system might have induced changes in ventilation which could account for the decrease in MSNA (expressed as total activity) and diastolic blood pressure observed during breathing room air with a mouthpiece compared with baseline values in our study. These changes are consistent with the increase in parasympathetic tone and the reflex decrease in sympathetic tone described during lung inflation (Seals et al., 1990). When the subjects inhaled cold air with a mouthpiece, sympathetic activation due to the cold stimulus seemed to override any indirect sympathoinhibitory effect due to altered ventilation, resulting in a mild to moderate increase in MSNA as a net effect.

The sympathetic and the parasympathetic system are closely linked in most cardiovascular reflexes. As we did not measure parasympathetic activity in our study, we are not able to demonstrate the parasympathetic origin of the bradycardia observed during cold exposure of the forehead and of the nasal mucosa.

\section{Conclusion}

Despite these limitations, our in vivo study in healthy human subjects demonstrates strong effects of facial cold exposure and cold air inhalation on the sympathetic nervous system and its cardiovascular effector organs. The sympathetic activation observed during the different cooling manoeuvres is probably mediated by cold-sensitive afferents in the skin or mucous membranes. The reflex pattern of facial cooling differs from the pattern of cold stress to the hand (CPT). Forehead cooling induces a stronger increase in MSNA but it decreases heart rate. Thus, activation of muscle sympathetic outflow most likely coincides with parasympathetic activation of the cardiac autonomic nervous system. This effect on the cardiac autonomic nervous system can also be seen when the cold stimulus is applied to the nasal cavity. Finally, sympathoexcitation is not restricted to external cold stimuli but can also be induced by inhalation of cold air.

\section{References}

Allen, M.T., Shelley, K.S., Boquet, A.J.J., 1992. A comparison of cardiovascular and autonomic adjustments to three types of cold stimulation tasks. Int. J. Psychophysiol. 13, 59-69.

Baile, E.M., Dahlby, R.W., Wiggs, B.R., Parsons, G.H., Paré, P.D., 1987. Effect of cold and warm dry air hyperventilation on canine airway blood flow. J. Appl. Physiol. 62, 526-532.

Delius, W., Hagbarth, K.E., Hongell, A., Wallin, B.G., 1972. General characteristics of sympathetic activity in human muscle nerves. Acta Physiol. Scand. 84, 65-81.

Dodt, C., Keyser, B., Mölle, M., Fehm, H.L., Elam, M., 2000. Acute suppression of muscle sympathetic nerve activity by hydrocortisone in humans. Hypertension 35, 758-763. 
Fagius, J., Sundlöf, G., 1986. The diving response in man: effects on sympathetic activity in muscle and skin nerve fascicles. J. Physiol. Lond. 377, 429-443.

Fagius, J., Karhuvaara, S., Sundlöf, G., 1989. The cold pressor test: effects on sympathetic nerve activity in human muscle and skin nerve fascicles. Acta Physiol. Scand. 137, 325-334.

Fontanari, P., Burnet, H., Zattara-Hartmann, M.C., Jammes, Y., 1996. Changes in airway resistance induced by nasal inhalation of cold dry, dry, or moist air in normal individuals. J. Appl. Physiol. 81, 1739-1743.

Hardy, J.C., Gray, K., Whisler, S., Leuenberger, U., 1994. Sympathetic and blood pressure responses to voluntary apnea are augmented by hypoxemia. J. Appl. Physiol. 77, 2360-2365.

Hayward, J.M., Holmes, W.F., Gooden, B.A., 1976. Cardiovascular responses in man to a stream of cold air. Cardiovas. Res. 10, 691-696.

Heindl, S., Lehnert, M., Criée, C.P., Hasenfuss, G., Andreas, S., 2001. Marked sympathetic activation in patients with chronic respiratory failure. Am. J. Respir. Crit. Care Med. 164, 597601.

Jones, A.S., Wight, R.G., Durham, L.H., 1989. The distribution of thermoreceptors within the nasal cavity. Clin. Otolaryngol. 14, 235-239.

Kregel, K.C., Seals, D.R., Callister, R., 1992. Sympathetic nervous system activity during skin cooling in humans: relationship to stimulus intensity and pain sensation. J. Physiol. Lond. 454, 359-371.

LeBlanc, J., Mercier, I., 1992. Cold wind stimulation reflex. J. Appl. Physiol. 73, 1704-1707.

Morgan, B.J., Crabtree, D.C., Palta, M., Skatrud, J.B., 1995. Combined hypoxia and hypercapnia evokes long-lasting sympathetic activation in humans. J. Appl. Physiol. 79, 205213.
Nordin, M., Fagius, J., 1995. Effect of noxious stimulation on sympathetic vasoconstrictor outflow to human muscles. J. Physiol. Lond. 489, 885-894.

Sant' Ambrogio, G., Mathew, O.P., Sant' Ambrogio, F.B., Fisher, J.T., 1985. Laryngeal cold receptors. Respir. Physiol. 59, 3544.

Seals, D.R., Suwarno, O., Dempsey, J.A., 1990. Influence of lung volume on sympathetic nerve activity in normal humans. Circ. Res. 67, 130-141.

Togias, A., Lykens, K., Kagey-Sobotka, A., Eggleston, P.A., Proud, D., Lichtenstein, L.M., Naclerio, R.M., 1990. Studies on the relationships between sensitivity to cold, dry air, hyperosmolal solutions, and histamine in the adult nose. Am. Rev. Respir. Dis. 141, 1428-1433.

Trouerbach, J., Duprez, D., De Buyzere, M., De Sutter, J., Clement, D., 1994. Cardiovascular responses elicited by different simulated diving manoeuvres. Eur. J. Appl. Physiol. Occup. Physiol. 68, 341-344.

Vallbo, A.B., Hagbarth, K.E., Torebjork, H.E., Wallin, B.G., 1979. Somatosensory, proprioceptive and sympathetic activity in human peripheral nerves. Physiol. Rev. 59, 919957.

Victor, R.G., Leimbach, W.N., Seals, D.R., Wallin, B.G., Mark, A.L., 1987. Effects of the cold pressor test on muscle sympathetic nerve activity in humans. Hypertension 9, 429436.

Walsh, J.T., Andrews, P., Batin, P.D., Cowley, A.J., 1995. Haemodynamic and hormonal response to a stream of cooled air. Eur. J. Appl. Physiol. 72, 76-80.

Widdicombe, J.G., 1986. The physiology of the nose. Clin. Chest Med. 7, 159-170.

Widdicombe, J., 2001. Airway receptors. Respir. Physiol. 125, 315. 